\title{
BIFURCATION ANALYSIS AND DYNAMIC BEHAVIOUR OF AN INVERTED PENDULUM WITH BOUNDED CONTROL*
}

\author{
Svetoslav Nikolov \\ Institute of Mechanics, Bulgarian Academy of Science, \\ Acad. G. Bonchev St., Bl. 4, 1113 Sofia, Bulgaria, \\ University of Transport, \\ 158, G. Milev St., 1574 Sofia, Bulgaria, \\ e-mail: s.nikolov@imbm.bas.bg \\ VALENTIN NEDEV \\ University of Transport, \\ 158, G. Milev St., 1574 Sofia, Bulgaria, \\ e-mail: val_nedev@abv.bg
}

[Received 05 January 2016. Accepted 29 February 2016]

\begin{abstract}
This paper presents an investigation on the behaviour of conventional inverted pendulum with an inertia disk in its free extreme. The system is actuated by means of torques applied to the disk by a DC motor, mounted on the pendulum's arm. Thus, the system is underactuated since the pendulum can rotate freely around its pivot point. The dynamical model is given with three ordinary nonlinear differential equations. Using Poincare-Andronov-Hopf's theory, we find a new analytical formula for the first Lyapunov's value at the boundary of stability. It enables one to study in detail the bifurcation behaviour of the above dynamic system. We check the validity of our analytical results on the first Lyapunov's value by numerical simulations. Hence, we find some new results.

KEY WORDS: Inverted pendulum, bounded control, bifurcation analysis, dynamic behaviour.
\end{abstract}

\section{Introduction}

Dynamical systems are families of motions, determined by evolutionary processes, the evolution of which takes place over time. These systems are

\footnotetext{
* Corresponding author e-mail:s.nikolov@imbm.bas.bg

This paper is funded by the grant No 1616/22.04.2014 of the University of Transport "T. Kableshkov" Sofia, Bulgaria.
} 
determined in a variety of ways. Perhaps, the most commonly encountered examples are those which are determined by models of first order ordinary differential equations. The term "dynamical system" first appears in mechanics, where it referred to a mechanical system with a finite number of degree of freedom.

For some critical parameters in the mechanical and mathematical models, significant changes in the properties of the solutions occur when they are modified around critical values called 'bifurcation points'. The dynamical properties of the investigated system can vary, when such modification happen and sustained oscillations can emerge from stable steady states or inversely, oscillations can disappear as damped time limited oscillations, that lead to steady states.

Bifurcation theory describes qualitative changes in phase portraits, that occur as parameters, are varied in the definition of a dynamical system [1]. The computation of Lyapunov quantities (values) is one of the central problems in considering of small limit cycles in the neighbourhood of equilibrium of two-, three-, and four-dimensional dynamical systems [2-7]. These values are also substantial in investigation of the different problems from engineering mechanics, when the corresponding dynamical systems are close to the boundary of stability domain of its parameters. According to [8], the safe (invertible) and dangerous (non-invertible) boundaries take place. Such boundaries correspond to scenario of "soft" and "hard" excitation of oscillations, which was firstly considered by Andronov [9].

Dynamic behaviour and stabilization of the inverted pendulum is one of the most interesting problems in modern nonlinear science [10, 11]. Here, we conduct an investigation of the dynamical behaviour of an inverted pendulum with bounded control (see Fig. 1). The system is of interest both from the point of view of dynamically rich structure that it possesses and also because it is fairly easy to construct a mechanical model of it.

Several investigators have sought to develop nonlinear dynamical models of the inverted pendulum $[12,13]$. It is found, that the inverted state stabilizes via alternating 'reverse' subcritical pitch-fork and period-doubling bifurcations, while it destabilizes via 'normal' supercritical period-doubling and pitch-fork bifurcations. On the other hand, in [14] Lozano et al. presented a stabilization of the inverted pendulum around its homoclinic orbit.

In the recent years, the research is focused towards obtaining control algorithms for general underactuated nonlinear mechanical systems. An Underactuated Mechanical System (UMS) is this which possesses fewer control inputs than degrees of freedom $[15,16]$. UMSs have been arosen in many 
different real-life applications, such as: helicopters, aircrafts, spacecrafts, vertical take-off and landing aircrafts, under water vehicles, mobile robots, walking robots and so on. In some UMSs, the lack of action on certain directions can be interpreted as constrains on the acceleration. Some of these systems represent academic benchmarks and are part of a standard control laboratory, like the inverted pendulum, the rotational inverted pendulum, the pendubot [17], the pendulum driven by a spinning wheel. Linear systems can be rendered passive via smooth state feedback and they extended a number of stabilization schemes for global asymptotic stabilization of certain classes of nonlinear systems. The main contribution of the above systems is to exploit their passivity properties to develop appropriate control laws [18].

\section{Problem formulation}

In order to balance the inverted pendulum at the upper equilibrium position, the control must eventually be switched to a controller, that guarantees (local) asymptotic stability of this equilibrium.

Here, we are interested in the dynamical behaviour of the inverted (inertial wheel) pendulum with bounded control. The system is actuated by means of torques applied to the disk by a DC motor, mounted on the pendulum's arm $[19,20]$. The system is underactuated since the pendulum can rotate freely around its pivot point - Fig. 1. In this case (according to [21]), the differential

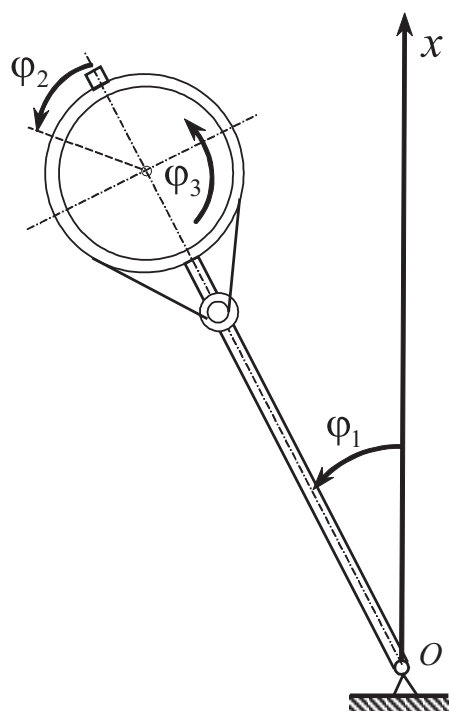

Fig. 1. The inverted underactuated pendulum 
equations of the motion of the pendulum have the form:

$$
\begin{aligned}
& \dot{\varphi}_{1}=\varphi_{2}, \\
& \dot{\varphi}_{2}=q_{1} \sin \varphi_{1}+q_{2} \varphi_{3}-q_{3} u, \\
& \dot{\varphi}_{3}=-q_{1} \sin \varphi_{1}-q_{2}(1+\rho) \varphi_{3}+q_{3}(1+\rho) u,
\end{aligned}
$$

where $\varphi_{1}$ is the angle of the $\operatorname{arm}\left(\varphi_{1}=0\right.$ at the upright position), $\varphi_{2}$ is the angular velocity of the arm, $\varphi_{3}$ is the angular velocity of the disk with respect to arm, $u$ is the control input (voltage applied to the motor), and $q_{1}, q_{2}, q_{3}$ and $\rho>0$ are constant coefficients, derived from physical parameters [21]. Here, we note that the disk position is not considered as a stable variable, because it is irrelevant for the stabilization of the pendulum in the inverted position.

The control objective is the stabilization of the inverted state of the pendulum, i.e. when the disk velocity is zero. Thus, according to [21], we consider an auxiliary control in the form:

$$
u=\alpha_{1} \sin \varphi_{1}+\alpha_{2} \varphi_{2}+\alpha_{3} \varphi_{3},
$$

where $\alpha_{1}, \alpha_{2}$ and $\alpha_{3}$ are control gains. It is well-know, that function $\sin \varphi_{1}$ can be written in Taylor series, i.e.:

$$
\sin \varphi_{1}=\varphi_{1}-\frac{1}{3 !} \varphi_{1}^{3}+\frac{1}{5 !} \varphi_{1}^{5}-\cdots
$$

If we take only the first and the second terms from (3), and after substitution of (2) into (1), Eq. (1) takes the form:

$$
\begin{aligned}
& \dot{\varphi}_{1}=\varphi_{2}, \\
& \dot{\varphi}_{2}=k_{1} \varphi_{1}-k_{2} \varphi_{2}+k_{3} \varphi_{3}-k_{4} \varphi_{1}^{3}, \\
& \dot{\varphi}_{3}=-k_{5} \varphi_{1}+k_{6} \varphi_{2}-k_{7} \varphi_{3}+k_{8} \varphi_{1}^{3},
\end{aligned}
$$

where:

$$
\begin{aligned}
k_{1} & =q_{1}-\alpha_{1} q_{3}, \quad k_{2}=\alpha_{2} q_{3}, \quad k_{3}=q_{2}-\alpha_{3} q_{3}, \quad k_{4}=\frac{1}{6}\left(q_{1}-\alpha_{1} q_{3}\right), \\
(5) \quad k_{5} & =q_{1}-\alpha_{1} q_{3}(1+\rho), \quad k_{6}=\alpha_{2} q_{3}(1+\rho), \quad k_{7}=(1+\rho)\left(q_{2}-\alpha_{3} q_{3}\right), \\
k_{8} & =\frac{1}{6}\left[q_{1}-\alpha_{1} q_{3}(1+\rho)\right] .
\end{aligned}
$$

These three dimensional nonlinear ordinary differential equations are autonomous (time does not appear explicitly).

The paper is organized as follows: in Section 3, we obtain the analytical results for first Lyapunov value, when control gains are varied. In Section 4, we present the numerical results. Finally, Section 5 summarizes our results.

\section{Qualitative analysis}

It is easy to see, that the equilibrium (steady states) values of the system (4) are:

$$
\bar{\varphi}_{1}^{(1)}=\bar{\varphi}_{2}^{(1)}=\bar{\varphi}_{3}^{(1)}=0,
$$


Bifurcation Analysis and Dynamic Behaviour of an Inverted Pendulum . . . 21

$$
\bar{\varphi}_{1}^{(2,3)}= \pm \sqrt{6}, \quad \bar{\varphi}_{2}^{(2,3)}=\bar{\varphi}_{3}^{(2,3)}=0 .
$$

We must accomplish some transformations of system (4), before determining analytically first Lyapunov value $\left(L_{1}\right)$ and Routh-Hurwitz conditions for stability of fixed points (6) and (7). First, we express (4) in the form:

$$
\dddot{\varphi}_{1}=-c_{1} \ddot{\varphi}_{1}+c_{2} \dot{\varphi}_{1}+c_{3} \varphi_{1}+c_{4} \varphi_{1}^{3}-c_{5} \varphi_{1}^{2} \dot{\varphi}_{1}
$$

where:

$$
\begin{aligned}
& c_{1}=k_{2}+k_{7}, \quad c_{2}=k_{1}+k_{3} k_{6}-k_{2} k_{7}, \\
& c_{3}=k_{1} k_{7}-k_{3} k_{5}, \quad c_{4}=k_{3} k_{8}-k_{4} k_{7}, \quad c_{5}=3 k_{4} .
\end{aligned}
$$

Let us denote:

$$
y=\varphi_{1}-\varphi_{0} .
$$

After substitution of (10) into (8), Eq. (8) takes the form:

$$
\begin{aligned}
\dddot{y}=-c_{1} \ddot{y}+\left(c_{2}-c_{5} \varphi_{0}^{2}\right) \dot{y}+\left(c_{3}+3 c_{4} \varphi_{0}^{2}\right) y \\
\quad-2 c_{5} \varphi_{0} y \dot{y}+3 c_{4} \varphi_{0} y^{2}+c_{4} y^{3}-c_{5} y^{2} \dot{y} .
\end{aligned}
$$

Here, $\varphi_{0}$ is the equilibrium states $\bar{\varphi}_{1}^{(2)}=\sqrt{6}$ or $\bar{\varphi}_{1}^{(3)}=-\sqrt{6}$. It is seen, that in linear terms of (11) we have $\varphi_{0}^{2}$, therefore, we can conclude that boundary of stability (and qualitative behaviour also) for $\bar{\varphi}_{1}^{(2,3)}$ will be one and the same.

On the other hand, let:

$$
\ddot{y}=w_{1}, \quad \dot{y}=w_{2}, \quad y=w_{3} .
$$

After substitution of (12) into (11), the third-order ordinary differential equation (11) is reduced to three first-order differential equations:

$$
\begin{aligned}
& \dot{w}_{1}=-c_{1} w_{1}+c_{6} w_{2}+c_{7} w_{3}-c_{8} w_{2} w_{3}+c_{9} w_{3}^{2}+c_{4} w_{3}^{3}-c_{5} w_{2} w_{3}^{2}, \\
& \dot{w}_{2}=w_{1}, \\
& \dot{w}_{3}=w_{2},
\end{aligned}
$$

where:

$$
\begin{aligned}
& c_{6}=c_{2}-c_{5} \varphi_{0}^{2}, \quad c_{7}=c_{3}+3 c_{4} \varphi_{0}^{2}, \\
& c_{8}=2 c_{5} \varphi_{0}, \quad c_{9}=3 c_{4} \varphi_{0} .
\end{aligned}
$$

The flow divergence (13) is:

$$
D_{3}=\frac{\partial \dot{w}_{1}}{\partial w_{1}}+\frac{\partial \dot{w}_{2}}{\partial w_{2}}+\frac{\partial \dot{w}_{3}}{\partial w_{3}}=-k_{2}-k_{7} .
$$

The system (13) is dissipative and has an attractor, when $D_{3}<0$, i.e. $\alpha_{2} \geq \alpha_{3}(1+\rho)$.

Following [8], the Routh-Hurwitz conditions for stability of (7) can be 
written in the form:

$$
\begin{aligned}
p & =c_{1}=k_{2}+k_{7}>0, \\
q & =-c_{6}=k_{2} k_{7}-k_{1}-k_{3} k_{6}+3 k_{4} \varphi_{0}^{2}>0, \\
r & =-c_{7}=-c_{3}-3 c_{4} \varphi_{0}^{2}>0, \\
R & =p q-r=-c_{1} c_{6}+c_{7}>0 .
\end{aligned}
$$

The notations $p, q, r$ and $R$ are taken from [8]. The boundaries of the stability region, according to $[2,8]$ for three dimensional dynamical system, are two surfaces: $\Psi_{1}(R=0, p>0, q>0)$ and $\Psi_{2}(r=0, p>0, q>0)$. On the surface $R=0$, the characteristic equation has a pair of purely imaginary roots and here, an Andronov-Hopf bifurcation takes place, and at least one zero root on the surface $r=0$.

From an analytical point of view, the onset of sustained oscillations generally corresponds to a transition through an Andronov-Hopf bifurcation point. Beyond the bifurcation point, a stable solution arises in the form of a small amplitude limit cycle, surrounding the unstable steady state. This bifurcation can be of two types: (i) supercritical (soft loss of stability) and (ii) subcritical (hard loss of stability).

When the condition (19) is not valid, the steady state (7) becomes unstable. In order to characterize the nature of the bifurcation points in the parameters $\alpha_{1}, \alpha_{2}$ and $\alpha_{3}$ of our model (13), the so-called first Lyapunov value, $L_{1}$, must be computed and analyzed for the system (13) $[2,3,8,9$, 22 . In the bifurcation points, a positive first Lyapunov value (this is not Lyapunov exponent- see appendix in [23] or for a detailed discussion [2, 9, $22]$ ), represents a hard (subcritical or irreversible) bifurcation and predicts, that the system presents unstable solutions, but may fold back and exhibit unstable periodic oscillations coexisting with stable steady states. In this case, the boundary of stability is considered as "dangerous". In contrast, a negative value for $L_{1}$ indicates a soft (supercritical or reversible) bifurcation. Here, the loss of stability takes place when stable self-oscillations emerge from a transition through the bifurcation point. In this case, the boundary of stability is considered "safe".

We summarize the main analytical results for first Lyapunov value here. Further details about the derivations of the formulae can be found in the Appendix. In the case of three first-order autonomous nonlinear differential equa- 
tions, this value can be determined analytically by the formula in [8]:

$$
\begin{aligned}
& L_{1}\left(\lambda_{0}\right)=\frac{\pi}{4 q}\left[2\left(A_{33}^{(2)} A_{33}^{(3)}-A_{22}^{(2)} A_{22}^{(3)}\right)+2 A_{23}^{(2)}\left(A_{22}^{(2)}+A_{33}^{(2)}\right)-\right. \\
& \left.\quad-2 A_{23}^{(3)}\left(A_{22}^{(3)}+A_{33}^{(3)}\right)+3 \sqrt{q}\left(A_{222}^{(2)}\right)+A_{333}^{(3)}+A_{233}^{(2)}+A_{223}^{(3)}\right]+ \\
& \quad+\frac{\pi}{4 p \sqrt{q}\left(p^{2}+4 q\right)}\left\{p ^ { 2 } \left[2 A_{22}^{(1)}\left(3 A_{12}^{(2)}+A_{13}^{(3)}\right)+2 A_{33}^{(1)}\left(A_{12}^{(2)}+3 A_{13}^{(3)}\right)+\right.\right. \\
& \left.\quad+4 A_{23}^{(1)}\left(A_{13}^{(2)}+A_{12}^{(3)}\right)\right]+4 p \sqrt{q}\left[\left(A_{22}^{(1)}-A_{33}^{(1)}\right)\left(A_{13}^{(2)}+A_{12}^{(3)}\right)+\right. \\
& \left.\left.\quad+2 A_{23}^{(1)}\left(A_{13}^{(3)}-A_{12}^{(2)}\right)\right]+16 q\left(A_{22}^{(1)}+A_{33}^{(1)}\right)\left(A_{12}^{(2)}+A_{13}^{(3)}\right)\right\}
\end{aligned}
$$

where $\lambda_{0}$ is defined as a value of $\alpha_{1}, \alpha_{2}, \alpha_{3}, q_{1}, q_{2}, q_{3}$ and $\rho$ for which the relation $R=0$ takes place. The coefficients $A_{i j}^{n}$ and $A_{i j k}^{n}(i, j, k, n=1,2,3)$ are defined by corresponding formulas, presented in [8]. According to [3, 8, 23], after accomplishing some transformations and algebraic operations, we obtain the formula describing the first Lyapunov value for the model investigated and calculate its value in the computed bifurcation points (for further details see Appendix).

We focus our analysis on the effect of changes on the bifurcation parameters $\alpha_{1}, \alpha_{2}$ and $\alpha_{3}$, which represent the control gains of the system. According to experiment work [21], we assume the following intervals of mechanically relevant values for $\alpha_{1}, \alpha_{2}$ and $\alpha_{3}: \alpha_{1} \in[15,400], \alpha_{2} \in[13,50], \alpha_{3} \in[0.1,0.5]$.

In Fig. 2, $L_{1}\left(\lambda_{0}\right)$ (calculated on the boundary of stability $R=0$ in Eq. (20); for further details, see Appendix) is shown for different values of the bifurcation parameters $\alpha_{1}, \alpha_{2}$ and $\alpha_{3}$. It can be seen that $L_{1}\left(\lambda_{0}\right)$ passes through regions for which it is negative or positive. Therefore, following the terms introduced in $[8,9]$, we have a soft and a hard stability loss. Unfortunately, Fig. 2 (left panel) gives only a most general idea about the change of $L_{1}$. A more detailed investigation of $L_{1}\left(\lambda_{0}\right)$ is shown in the following Fig. 2 (right panel). What could one observe in the figure? Practically, for different values of the parameter $\alpha_{2}$ we have different kind boundary of stability. For example, the boundary of stability $R=0$ (when $\alpha_{2}=13$ and $\alpha_{2}=50$ ) passes through different regions of $\left(\alpha_{1}, \alpha_{3}\right)$ space. In other words, different kind of dynamical behaviour is possible. Hence, we can conclude, that the analysis via Lyapunov-Andronov theory and first Lyapunov value helps us describe and predict the qualitative behaviour of the system for values of $\alpha_{1}, \alpha_{2}$ and $\alpha_{3}$, higher or smaller than the critical values, established approximated intervals of values for $\alpha_{1}, \alpha_{2}$ and $\alpha_{3}$ in which self-oscillations of the system occur (disappear). However, the precise features of such solutions must be investigated via numerical simulation. 
Here, we note, that the bifurcation theory of control systems focuses on the change of its qualitative properties, such as controllability, stabilizability and the topology of the equilibrium set. In this paper, the second problem is generally investigated. In particular, it is seen in Fig. 2, that only in some bounded (but large) control parameter intervals the limit cycle is stable, i. e. the control of oscillations is bounded effective.

In summary, the results obtained for first Lyapunov value (see Fig. 2) are key because we have defined a computable, finite region of the $\alpha_{1}-\alpha_{2}-\alpha_{3}$ space in which sustained oscillations can occur after "soft" or "hard" stability loss and in which we can search for oscillatory solutions. In the following section, we demonstrate numerically different types of behaviour for some particular values of the model parameters.

\section{Numerical simulations}

In this section, we perform a numerical analysis of the model (4), based on the our previous analytical results obtained in Section 3. The parameter values used in the numerical calculations were selected according to [21], i. e.
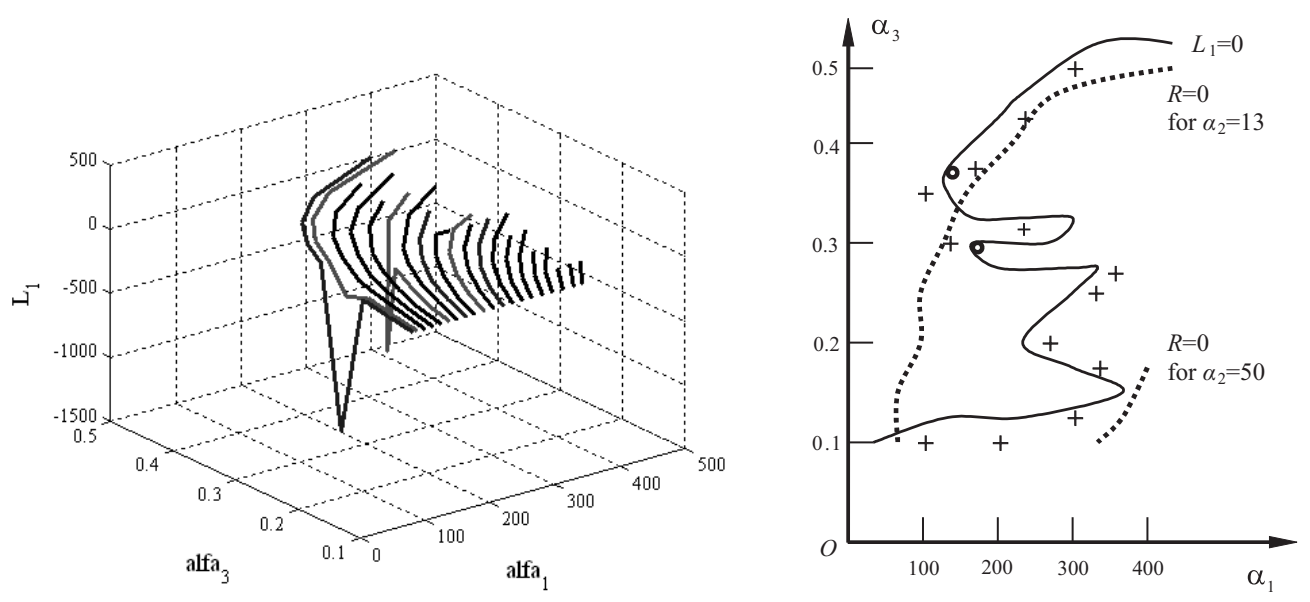

Fig. 2. Results for first Lyapunov value $L_{1}$ as function of parameters $\alpha_{1} \in$ $[15,400], \alpha_{2} \in[13,50]$ and $\alpha_{3} \in[0.1,0.5]$. It is seen that boundary of stability $R=0$ can be 'safe' and 'dangerous' because $L_{1}$ is negative or positive. Here we note that the another system parameters are fixed and are: $q_{1}=30, q_{2}=0.0245, q_{3}=0.0393$, $\rho=250$ 
we used the following values for the system parameters:

$$
\begin{array}{lll}
q_{1}=30\left[\mathrm{~s}^{-2}\right], & q_{2}=0.0245\left[\mathrm{~s}^{-1}\right], & q_{3}=0.0393\left[\mathrm{~s}^{-1}\right], \\
\rho \in[249.4,250.1], & & \\
\alpha_{1} \in[15,400]\left[\mathrm{s}^{-1}\right], & \alpha_{2} \in[13,50], & \alpha_{3} \in[0.1,0.5] .
\end{array}
$$

Here, we note that $\alpha_{2}, \alpha_{3}$ and $\rho$ are dimensionless parameters and in all our simulations time is in seconds. In Fig. 3, the stable solutions for the angle of the arm, $\varphi_{1}[\mathrm{rad}]$, the angular velocity of the arm, $\varphi_{2}\left[\mathrm{~s}^{-1}\right]$, and the angular velocity of the disk with respect to arm, $\varphi_{3}\left[\mathrm{~s}^{-1}\right]$, are shown for $\alpha_{1}=75, \alpha_{2}=30$ and $\alpha_{3}=0.5$. It is evident, that after several physically acceptable fluctuations (see Fig. $3 \mathrm{a}, \mathrm{b}, \mathrm{c}), \varphi_{1}, \varphi_{2}$ and $\varphi_{3}$ approach constant values (equilibrium state). In other words, in this case the conditions (16)-(19) from previous section are satisfied and the system (4) lies in stable zone of its parametric space. Here, we note that the governing equations of the model, represented by (4), were solved numerically using MATLAB [24] with initial
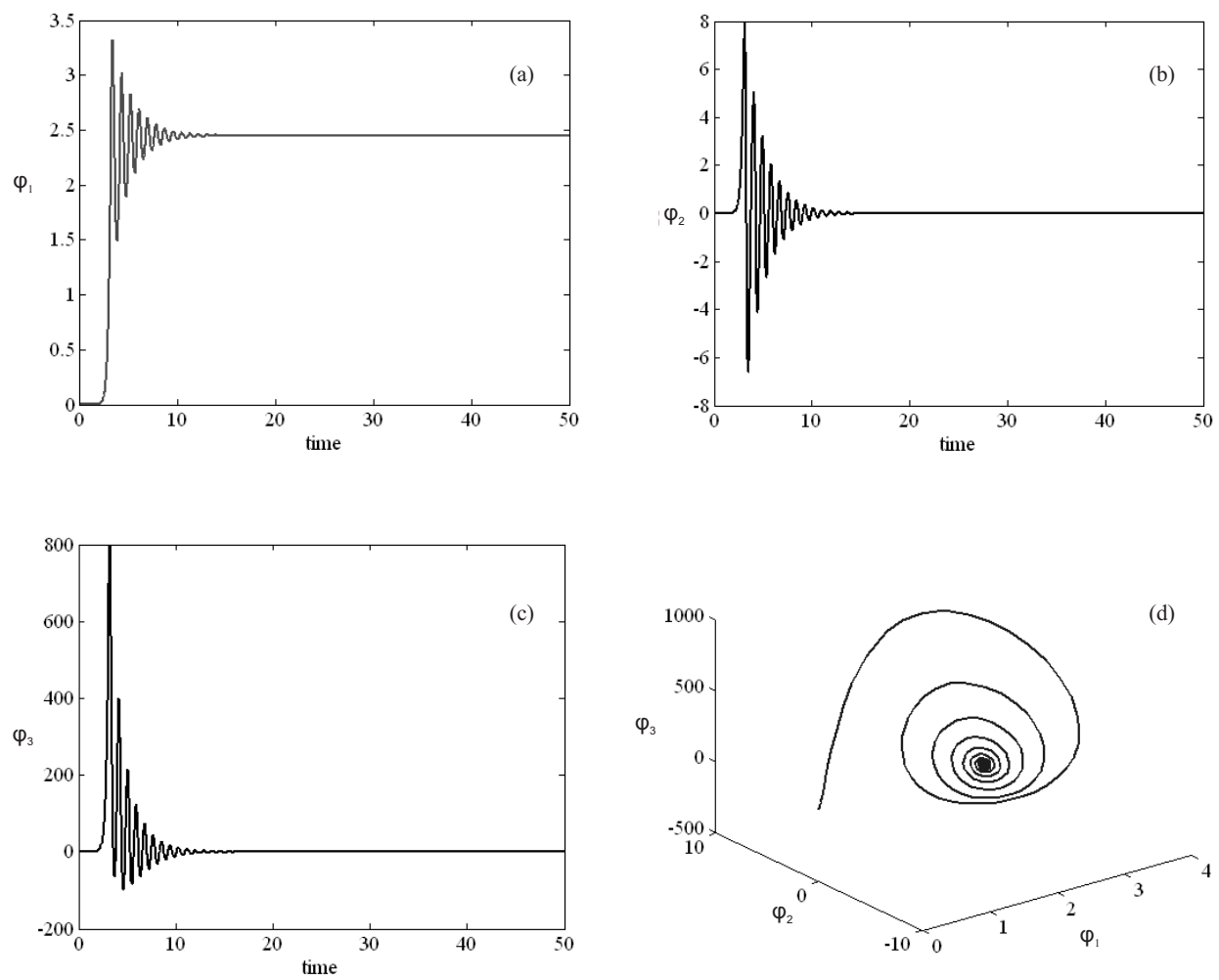

Fig. 3. Stable solutions and phase space of system (4). Time is in seconds 
conditions $\varphi_{1}^{0}=\varphi_{2}^{0}=0, \varphi_{3}^{0}=0.01$. In Fig. $3 \mathrm{~d}$, the phase space of the system (4) is shown.

In the next Fig. 4, the periodic solutions and the phase space of the system (4) are shown. In this case, the following values of the model parameters are accepted: $q_{1}=30, q_{2}=0.0245, q_{3}=0.0393, \rho=250, \alpha_{1}=376, \alpha_{2}=30$, $\alpha_{3}=0.5$. Our simulation demonstrates that the pendulum inverse state is stabilized near equilibrium (6) and a homoclinic limit cycle occurs. The analysis of the results suggests that in this case the system possesses one unstable fixed point from kind saddle and two stable fixed points from kind focus. Here, we note that these results are in accordance with the analytical results obtained in previous Section 2 and those in [14, 21].

Figure 5 illustrates how the system (4) changes its dynamic behaviour as function of first Lyapunov value. Figure 5 a depicts the solution of the system
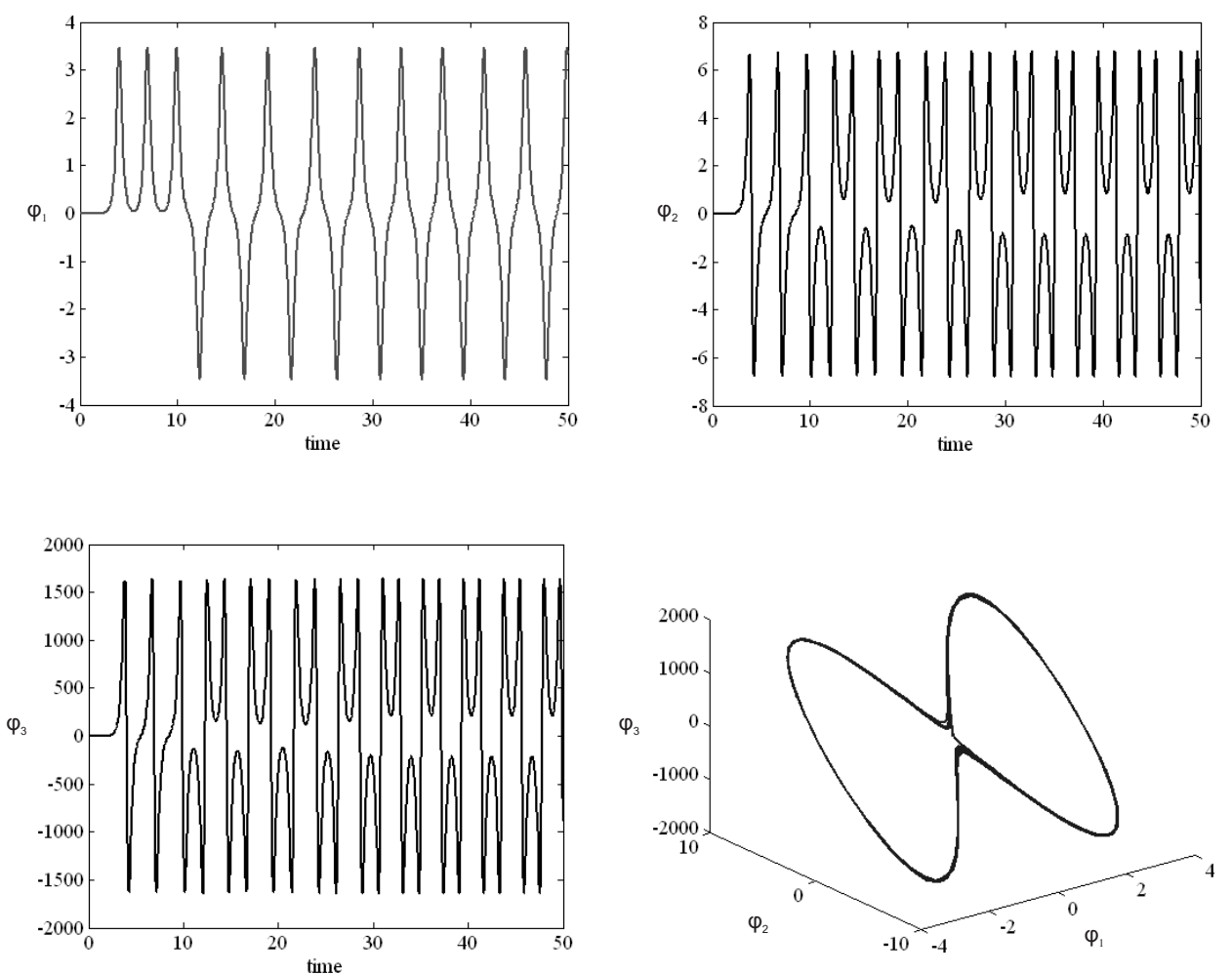

Fig. 4. Periodic solution and phase portrait of system (4). Time is in seconds. For details see text 

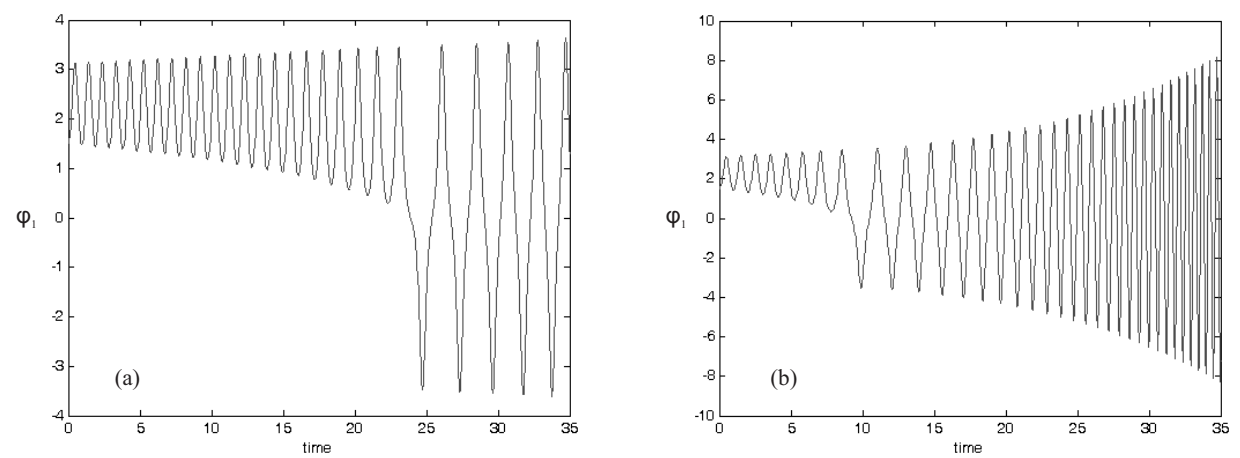

Fig. 5. (a) Solution of system (4) when the boundary of stability is 'safe'. Here $\alpha_{1}=89.03, \alpha_{2}=13.387$ and $\alpha_{3}=0.15$; (b) Solution of system (4) when the boundary of stability is 'dangerous'. Now $\alpha_{1}=159, \alpha_{2}=13.387$ and $\alpha_{3}=0.35$. For details see text

(4) in the case when the boundary of stability is 'safe', i. e. $L_{1}\left(\lambda_{0}\right)<0$. It is evident, that stable limit cycle with small amplitude occurs. Here, we note that system (4) in this case has reversible behaviour, i. e. a slow drift of the parameters back into the stability region brings a system back into the original response. On the other hand, Fig. 5b shows the case when the boundary of stability is dangerous (i. e. $L_{1}\left(\lambda_{0}\right)>0$ ). This is the case when the system undergoes very fast to large fluctuations and transition through the stability boundary may result in disappearance of the equilibrium state. Therefore, the system is structurally unstable (un-robust).

\section{Conclusions}

The proposed study treats the problem of dynamical behaviour of a conventional inverted pendulum with an inertia disk in its free extreme is considered. The system is actuated by means of torques applied to the disk by a DC motor, mounted on the pendulum's arm. The system is underactuated since the pendulum can rotate freely around its pivot point. The results found in the present study are in broad agreement with those of [21]. However, some additional dynamical behaviours of the inverted pendulum were shown.

The investigation of this paper is concerned with the question of whether the change of control gains $\alpha_{1}, \alpha_{2}$ and $\alpha_{3}$ alters the dynamic properties (stabilization) of an inverted pendulum with bounded control. General conclusions about design principles and the role of control gains can be suggested, since this system is a classical case study, covering several essential features of inverted 
pendulum and nonlinear control mechanisms

Our investigation is devoted to the use of the bifurcation analysis [1, $2,9]$, as a useful tool to investigate such state transitions when strong perturbation in the system parameters occurs. In a bifurcation point for a given parameter, the dynamical system is always structurally unstable and the stability conditions of limit cycles depend critically on stability conditions of steady states. We are able to define not only the structural stability (robustness) of the steady state, but also the robustness of limit cycles or other types of trajectories, by knowing the sign of the first Lyapunov value at the bifurcation boundary, as we showed in this work. This is a very central point of the bifurcation and stability analysis of steady states, periodic orbits and other types of trajectories. In our case, Lyapunov-Andronov theory were employed for the investigation of the original system (4) and the equivalent system (13). Hence, a new major conclusion of our analysis is the fact that in finite region of the $\alpha_{1}-\alpha_{2}-\alpha_{3}$ space the system (4) can have both "soft" and "hard" stability loss, as boundary of stability in first case is "safe" and in second one "dangerous", i. e. reversible and unreversible loss of stability take place.

As a result of our analysis, we found also that in the model (4) may induce the emergence of system configurations with two stable focuses and an unstable saddle point of first order. Under these conditions, the system displays bistability with homoclinic sustained oscillations. In our analysis, we have found at least one possibility to recover bistability; for details see Figs 6-8 in Appendix.

\section{REFERENCES}

[1] Guckenheimer, J., P. Worfolk. Dynamical Systems: Some Computational Problems, In: Bifurcations and Periodic Orbits of Vector Fields, London, Kluwer Academic Publishers, 1992, 241-279.

[2] Shilnikov, L., A. Shilnikov, D. Turaev, L. Chua. Methods of Qualitative Theory in Nonlinear Dynamics, Part I and II, London, World Scientific, 2001.

[3] Nikolov, S. First Lyapunov Value and Bifurcation Behaviour of Specific Class Three-dimensional Systems. Int. J. of Bifurcation and Chaos, 14 (2004), No. 8, $2811-2823$.

[4] Kuznetsov, Yu. Elements of Applied Bifurcation Theory, Second Edition, New York, Springer, 1998.

[5] Leonov, G., O. Kuznetsov. Lyapunov Quantities and Limit Cycles of Twodimensional Dynamical Systems. Analytical Methods and Symbolic Computation. Regular and Chaotic Dynamics, 15 (2010), No. 2-3, 354-377. 
Bifurcation Analysis and Dynamic Behaviour of an Inverted Pendulum . . 29

[6] Nikolov, S., V. Kotev, E. Yankulova. Bifurcation and Dynamical Behaviour of a Mathematical Model of HIV Infection. J. of Theoretical and Applied Mechanics, 37 (2007), No. 2, 101-116.

[7] Starkov, K. On the Ultimate Dynamics of the Four-dimensional Rossler System. Int. J. of Bifurcation and Chaos, 24 (2014), No. 11, art. No. 1450149 (7 pages).

[8] Bautin, N. Behaviour of Dynamical Systems near the Boundary of Stability, Moscow, Nauka, 1984.

[9] Andronov, A., A. Vitt, S. Khaikin. Theory of Oscillations, MA, Reading, Addison-Wesley, 1966.

[10] Sagdeev, R., D. Usikov, G. Zaslavsky. Nonlinear Physics: From the Pendulum to Turbulence and Chaos, Chur, Harwood Acad. Publ., 1992.

[11] Muskinja, N., B. Tovirnik. Swinging up and Stabilization of a Real Inverted Pendulum. IEEE Transactions on Industrial Electronics, 53 (2006), No. 2, 631639.

[12] KIm, S., B. Hu. Bifurcations and Transitions to Chaos in an Inverted Pendulum. Physical Review E, 58 (1998), No. 3, 3028-3035.

[13] Nikolov, S., S. Bachvarov. Dynamical Behaviour of Inverted Pendulum with a Cycloidal Oscillating Suspension Point. Engineering Mechanics, 11 (2004), No. 3, 201-214.

[14] Lozano, R., I. Fantoni, D. Block. Stabilization of the Inverted Pendulum Around its Homoclinic Orbit. Systems \& Control Letters, 40 (2000), No. 3, 197204.

[15] Andary, S., A. Chemori, S. Krut. Stable Limit Cycle Generation for Underactuated Mechanical Systems. Application: Inertia Wheel Inverted Pendulum, In: IROS'08: EEE/RSJ International Conference on Intelligent Robots and Systems, France, Nice, 2008.

[16] Braham, A., B. Cherki, M. Duemai, K. Busawon. Analysis and Control of Underactuated Mechanical Systems, NY, Springer, 2014.

[17] Spong, M., D. Block. The Pendubot: a Mechatronic System for Control Research and Education. In: Proc. of $34^{\text {th }}$ IEEE Conf. on Decision and Control, New Orleans, 1 (1995), 555-556.

[18] Fantoni, I., R. Lozano. Nonlinear Control for Underactuated Mechanical Systems, London, Springer-Verlag, 2002.

[19] Spong, M., P. Corke, R. Lozano. Nonlinear Control of the Inertia Wheel Pendulum. Automatica, 37 (1996), 1845-1851.

[20] Block, D., K. Astrom, M. Spong. The Reaction Wheel Pendulum. Synthesis Lectures on Control and Mechatronics, 1 (2007), No. 1, 1-105.

[21] Alonso, D., E. E. Paolini, J. Moiola. Controlling an Inverted Pendulum with Bounded Controls, In: Dynamics, Bifurcations, and Control, London, SpringerVerlag, 2002, 3-16.

[22] Leonov, G. A., N. V. Kuznetsov. Hidden Attractors in Dynamical Systems. From Hidden Oscillations in Hilbert-Kolmogorov, Aizerman, and Kalman Problems to Hidden Chaotic Attractor in Chua Circuits. Int. J. of Bifurcation and Chaos, 23 (2013), No. 1, art. No 1330002 (69 pages). 
[23] Nikolov, S., V. Petrov. New Results about Route to Chaos in Rossler System. Int. J. Bifurcation and Chaos, 14 (2004), No. 1, 293-308.

[24] MATLAB, The MathWorks Inc., MA, Natick, 2010, http://mathworks.com/.

[25] Seifert, H., W. Threlfall. Variationsrechnung im Groszen (Morsesche Theorie), Berlin, Teubner, 1938.

[26] Gammaitoni, L., I. Neri, H. Vocca. Nonlinear Oscillators for Vibration Energy Harvesting. Applied Physics Letters, 94 (2009), No. 16, art. No 164102 (3 pages).

\section{Appendix}

1. Calculation of the first Lyapunov value

In this paper, following [8], we calculate the first Lyapunov value. Generally in accordance with Lyapunov-Andronov theory, we have the following: (i) the sign of Lyapunov's value determines the character (stable or unstable) of equilibrium state at $R=0$; (ii) the character of equilibrium state at $R=0$ qualitatively determines the reconstruction of phase space (including stability or instability of limit cycle) at the transition from $R<0$ to $R>0$.

After accomplishing some transformations and algebraic operations for the coefficients $A_{i j}^{n}$ and $A_{i j k}^{n}(i, j, k, n=1,2,3)$ (into (20)), we obtain the following for the system (13):

$$
A_{33}^{(n)}=A_{333}^{(3)}=A_{233}^{(2)}=0, \quad A_{22}^{(n)}=\frac{1}{\Delta_{0}} \alpha_{n 1}^{\prime} a_{33}^{(1)}, \quad A_{23}^{(n)}=\frac{1}{\Delta_{0}} \alpha_{n 1}^{\prime} \alpha_{23} a_{23}^{(1)},
$$

$$
A_{12}^{(n)}=\frac{1}{\Delta_{0}} \alpha_{n 1}^{\prime}\left(a_{33}^{(1)} \alpha_{31}+a_{23}^{(1)} \alpha_{21}\right), \quad A_{13}^{(n)}=\frac{1}{\Delta_{0}} \alpha_{n 1}^{\prime} \alpha_{23} \alpha_{31} a_{23}^{(1)},
$$

$A_{222}^{(2)}=\frac{1}{\Delta_{0}} \alpha_{21}^{\prime} a_{333}^{(1)}, \quad A_{223}^{(3)}=\frac{1}{\Delta_{0}} \alpha_{31}^{\prime} \alpha_{23} a_{233}^{(1)}$.

Here, we note that:

$$
\begin{aligned}
& \alpha_{11}=p c_{7}, \quad \alpha_{21}=c_{7}, \quad \alpha_{31}=p\left(p-c_{4}\right)-c_{6}, \\
& \alpha_{12}=c_{6}, \quad \alpha_{32}=1, \quad \alpha_{23}=-\sqrt{q}, \quad \alpha_{22}=\alpha_{13}=\alpha_{33}=0, \\
& a_{23}^{(1)}=-c_{8}, \quad a_{33}^{(1)}=c_{9}, \quad a_{333}^{(1)}=c_{4}, \quad a_{233}^{(1)}=-c_{5},
\end{aligned}
$$

and

$$
\Delta_{0}=\operatorname{det}\left|\begin{array}{ccc}
\alpha_{11} & \alpha_{12} & \alpha_{13} \\
\alpha_{21} & \alpha_{22} & \alpha_{23} \\
\alpha_{31} & \alpha_{32} & \alpha_{33}
\end{array}\right|=\alpha_{12} \alpha_{23} \alpha_{31}-\alpha_{11} \alpha_{23}
$$


Bifurcation Analysis and Dynamic Behaviour of an Inverted Pendulum ... 31

From the previous formula (A.3), we obtain that:

$$
\alpha_{11}^{\prime}=\alpha_{23}, \quad \alpha_{21}^{\prime}=-\alpha_{23} \alpha_{31}, \quad \alpha_{31}^{\prime}=\alpha_{21} .
$$

\section{Numerical results}

Here, we analyze numerically the model constituted by (4). The governing equations of the model were solved numerically using MATLAB [17]. The corresponding numerical values of the model parameters are those in (21). The following Figs 6-8 demonstrate the dependence of the model behaviour on the parameter $\alpha_{1}$, when the another model parameters are fixed, i.e. $\alpha_{2}=30.387$, $\alpha_{3}=0.2, q_{1}=30, q_{2}=0.0245, q_{3}=0.0393, \rho=250$. In other words these
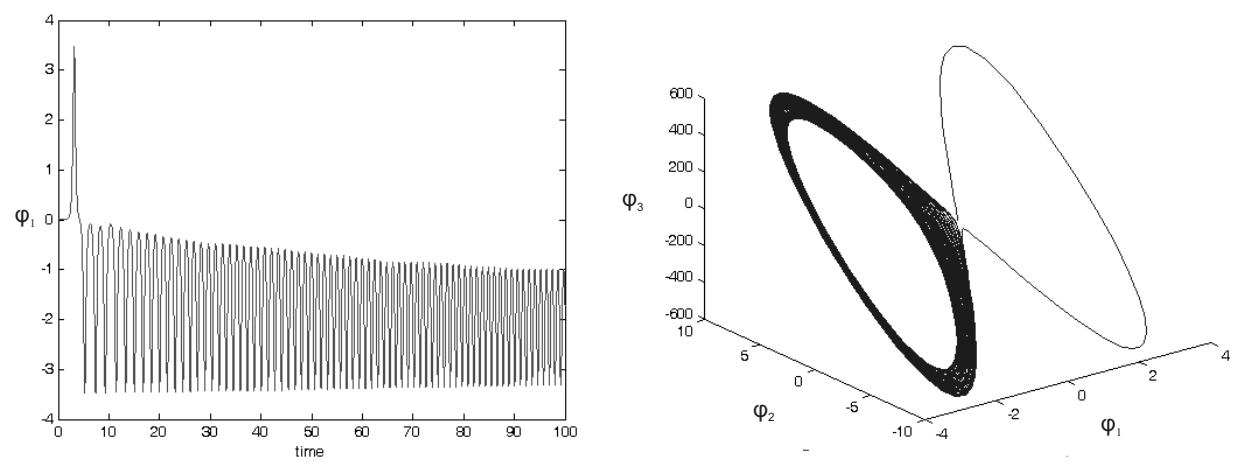

Fig. 6. Stable solution of system (4) near fixed point with coordinate $\bar{\varphi}_{1}=-\sqrt{6}$ when $\alpha_{1}=169.3, \alpha_{2}=30.387$ and $\alpha_{3}=0.2$. For more details see text
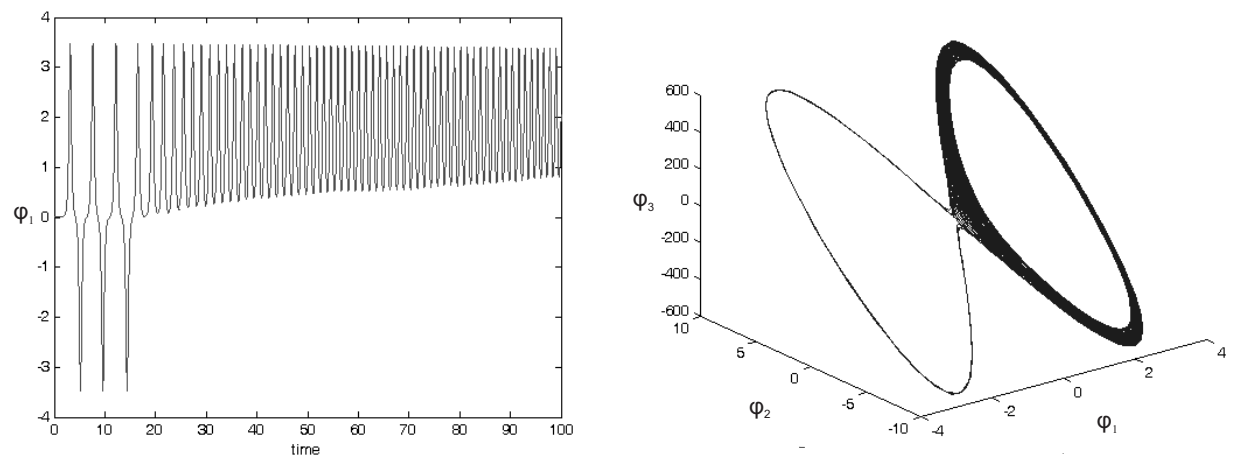

Fig. 7. Stable solution of system (4) near fixed point with coordinate $\bar{\varphi}_{1}=-\sqrt{6}$ when $\alpha_{1}=169.5, \alpha_{2}=30.387$ and $\alpha_{3}=0.2$. For more details see text 

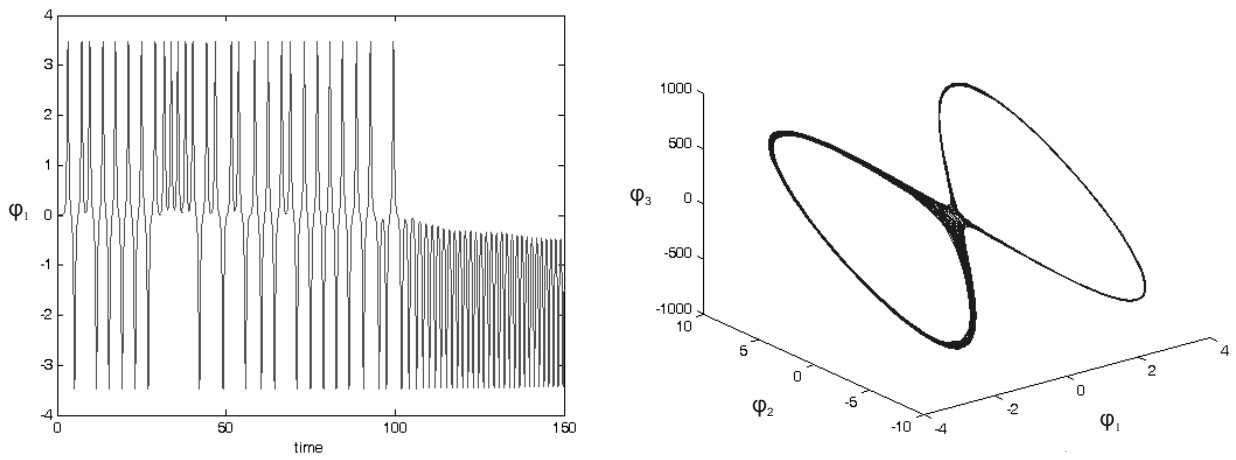

Fig. 8. Stable solution of system (4) near fixed point with coordinate $\bar{\varphi}_{1}=-\sqrt{6}$ with very long oscillation-like transient regime. Here, $\alpha_{1}=169.8, \alpha_{2}=30.387$ and $\alpha_{3}=0.2$. For more details see text

figures illustrate how the solution of the system (4) changes when parameter $\alpha_{1}$ is equal to $169.3,169.5$ and 169.8. It is seen, that for first and second value the system has a stable solution, which is near different fixed point with coordinate $\bar{\varphi}_{1}=-\sqrt{6}$ and $\bar{\varphi}_{1}=\sqrt{6}$. Under these conditions, the system displays bistability. It can be seen also that in Fig. 8 the oscillation-like transient regime is very long. Therefore, in this case the system lies very close to the boundary region, between two stable zones of attraction of fixed points $(7)[18,19]$. Here, we note that similar behaviour can be seen also for another values of $\alpha_{1}$ in the interval from 15 to 400 . 